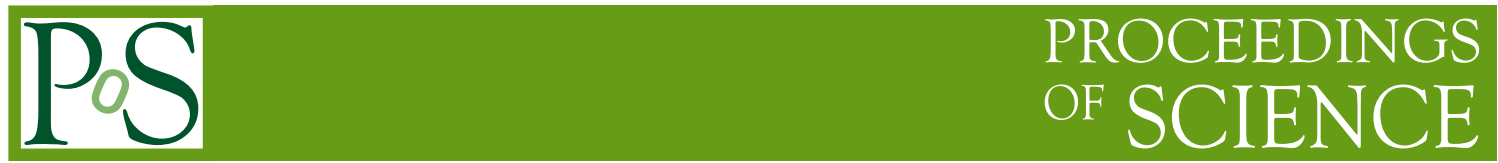

\title{
Phase Quenched Lattice QCD at Finite Density and Temperature
}

\section{K. Sinclair*}

HEP Division, Argonne National Laboratory, 9700 South Cass Ave., Argonne, IL, 60439, USA

E-mail: dks@hep.anl.gov

\section{J. B. Kogut}

Department of Energy, Division of High Energy Physics, Washington, DC 20585, USA

and

Dept. of Physics - TQHN, Univ. of Maryland, 82 Regents Dr., College Park, MD 20742, USA

E-mail: jbkogut@umd.edu

We simulate 3-flavour lattice QCD at finite quark-number chemical potential $\mu$ in the phasequenched approximation, close to the finite temperature transition. Working close to the critical quark mass, we find no evidence for the expected critical endpoint at small $\mu$. We are performing further simulations aimed at calculating the equation-of-state of this theory outside of the superfluid domain, where its phase structure is expected to mimic the full theory.

The XXV International Symposium on Lattice Field Theory

July 30 - August 42007

Regensburg, Germany

\footnotetext{
*Speaker.
} 


\section{Introduction}

QCD at finite chemical potential $\mu$ has a complex fermion determinant, which prevents direct application of the standard simulation methods of lattice QCD. We adopt the phase-quenched approximation, where we replace the fermion determinant by its magnitude, which allows the use of standard simulation methods. Outside of the superfluid phase in the $(T, \mu)$ plane, it is likely that the phase structures of full and phase-quenched QCD are the same. We use exact RHMC simulations [1], employing a speculative lower bound to the spectrum of the Dirac operator to enable the required use of rational approximations [2].

We simulate 3-flavour lattice QCD on $8^{3} \times 4,12^{3} \times 4$ and $16^{3} \times 4$ lattices for masses near to the critical mass $m_{c}$ at $\mu=0$, for temperatures close to the transition temperature from hadronic matter to a quark-gluon plasma. If the critical mass increases with increasing $\mu$, then for masses just above $m_{c}(0)$ we would find a critical endpoint at $m=m_{c}(\mu)$ for small $\mu$. However, as our simulations indicate, $m_{c}$ decreases with increasing $\mu$ and no critical endpoint is found for small $\mu$ [3]. We introduce $\mu_{I}=2 \mu$, which has the interpretation of an isospin chemical potential (at least for even numbers of flavours).

We are now simulating this same 3-flavour lattice QCD for a range of $\mu$ and $\beta$ values outside the superfluid phase, in order to calculate the equation-of-state for phase-quenched QCD.

\section{Simulations and results}

The nature of the transition is best determined on finite lattices using the 4th-order Binder cumulant for the magnetic order parameter. Since, at finite quark mass, we do not know this order parameter, we use the chiral condensate, introducing (hopefully small) finite size effects. The Binder cumulant for any observable $X$ is defined by [4]

$$
B_{4}(X)=\frac{\left\langle(X-\langle X\rangle)^{4}\right\rangle}{\left\langle(X-\langle X\rangle)^{2}\right\rangle^{2}}
$$

If there is a critical endpoint at small $\mu_{I}$ for $m>m_{c}(0)$, then the Binder cumulant should decrease from its crossover value $B_{4}=3$, passing through the Ising value $B_{4}=1.604(1)$ at the endpoint, and falling towards its first-order value $B_{4}=1$ as $\mu_{I}$ is increased. Small $\mu_{I}$ means $\mu_{I}$ small enough to lie outside of the superfluid phase. For $T=0$, this means $\mu_{I}<m_{\pi}$.

Since, as determined in these simulations, $m_{c}\left(\mu_{I}=0\right)=0.0265(3)$, we perform simulations at quark mass $m=0.02, m=0.025, m=0.03$ and $m=0.035$. At the 3 larger masses we simulate at $\mu_{I}=0, \mu_{I}=0.2, \mu_{I}=0.3$, while for $m=0.02$ we only simulate at $\mu_{I}=0$. On the $12^{3} \times 4$ lattice where we have the highest statistics, we generate 300,000 trajectories at each of $4 \beta$ values close to $\beta_{c}$ (the transition value), and use Ferrenberg-Swendsen reweighting [5] to continue to $\beta_{c}$, taken as the $\beta$ which minimizes $B_{4}(\bar{\psi} \psi)$. Five noisy estimators of $\bar{\psi} \psi$ per trajectory are used to obtain an unbiased estimator for $B_{4}$.

Figure 1 shows these Binder cumulants as functions of $\mu_{I}$ for $m=0.03$ on $8^{3} \times 4,12^{3} \times 4$ and $16^{3} \times 4$ lattices. Rather than decrease with increasing $\mu_{I}$, the curves for the larger lattices show a modest increase with increasing $\mu_{I}$, and hence no sign of a critical endpoint. The graphs for $m=0.035$ are similar, except that we do not have 'data' on $16^{3} \times 4$ lattices. At $m=0.025$, although there is a suggestion of such an increase, but the 'data' is consistent with no $\mu_{I}$ dependence. 


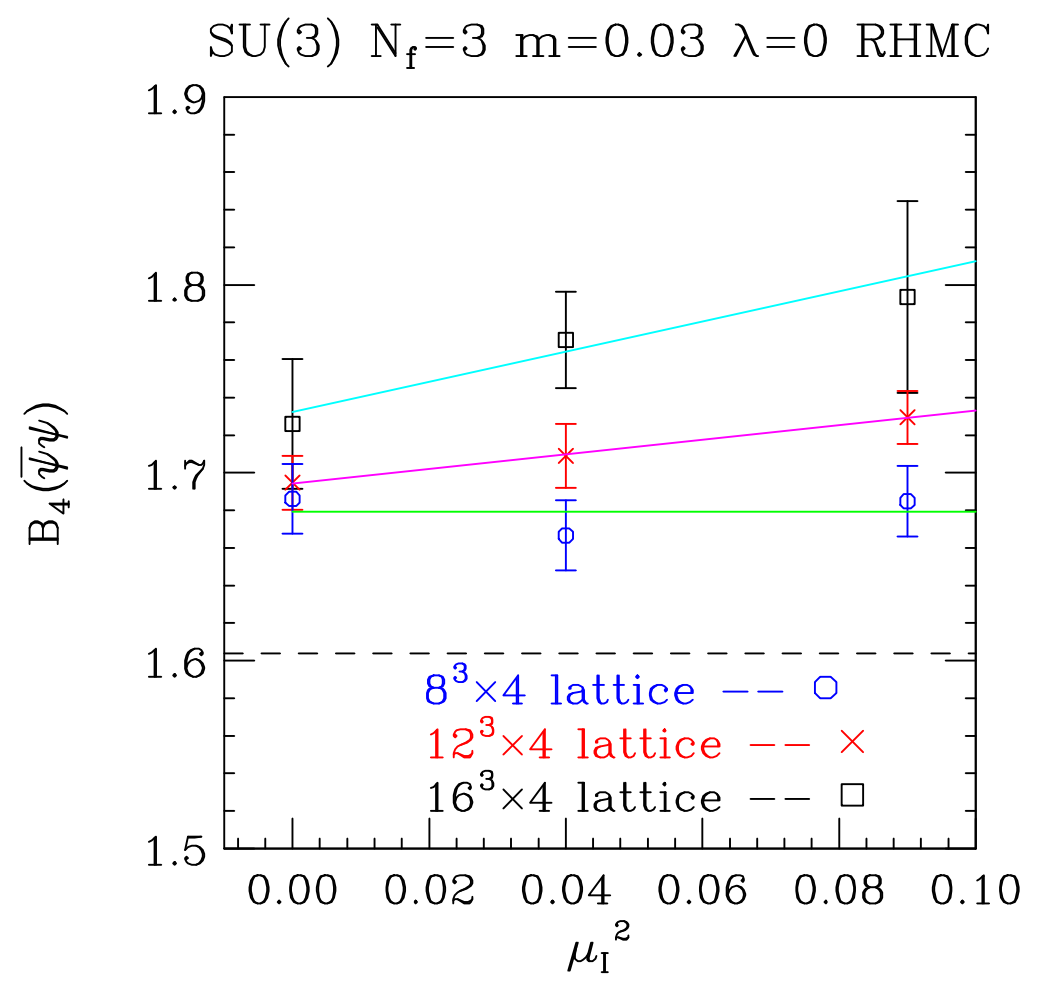

Figure 1: Binder cumulants at $T=T_{c}$ as a functions of $\mu_{I}^{2}$ at $m=0.030$.

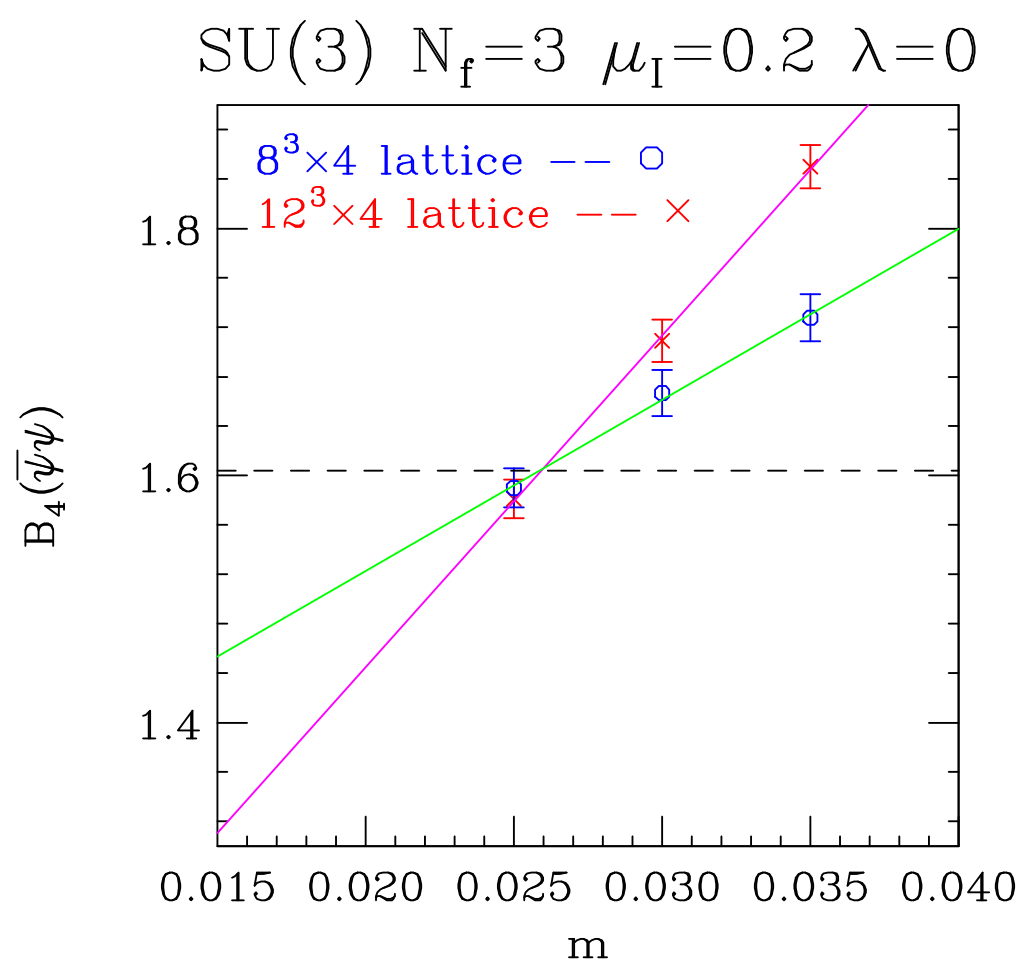

Figure 2: Binder cumulants at $T=T_{c}$ as a functions of $m$ at $\mu_{I}=0.2$. 
It is instructive to examine the behaviour of $B_{4}$ as functions of $m$ at fixed $\mu_{I}$ for evidence of a critical point in the universality class of the 3-dimensional Ising model. We find that for each of our $3 \mu_{I}$ values the curves for the $8^{3} \times 4$ and $12^{3} \times 4$ lattices cross very close to the Ising value. Not only does this indicate that the critical point belongs to the Ising universality class, but also suggests that using $\bar{\psi} \psi$ as the magnetic order parameter is a reasonable choice. Figure 2 shows $B_{4}$ as functions of $m$ for $\mu_{I}=0.2$. Estimating the position of the critical points as the values of $m$ where the $12^{3} \times 4$ curves achieve their Ising value gives: $m_{c}(0)=0.0265(3), m_{c}(0.2)=0.0259(5)$ and $m_{c}(0.3)=0.0256(4)$, i.e. $m_{c}\left(\mu_{I}\right)$ is a slowly decreasing function of $\mu_{I}$.

From the same 'data' we have also calculated the chiral susceptibility

$$
\chi_{\bar{\psi} \psi}=\frac{V}{T}\left\langle\left\langle\bar{\psi} \psi^{2}\right\rangle-\langle\bar{\psi} \psi\rangle^{2}\right\rangle
$$

where the $\bar{\psi} \psi$ s on the right-hand side are lattice averaged quantities. Finite size scaling at the critical point predicts that

$$
\chi_{\bar{\psi} \psi}\left(L, T_{c}\right)=L^{\frac{\gamma}{v}} \tilde{\chi} .
$$

Hence if we plot $L^{-\frac{\gamma}{v}} \chi_{\bar{\psi} \psi}\left(L, T_{c}\right)$ as functions of $m$ for different $L$ s, the curves should intersect at the critical point. In figure 3 we plot this quantity for $\mu_{I}=0.2$, and note that curves for $8^{3} \times 4$ and $12^{3} \times 4$ lattices would intersect between $m=0.025$ and $m=0.03$ which is where we found the critical point from the Binder cumulants. Similar results obtain for $\mu_{I}=0$ and $\mu_{I}=0.3$.

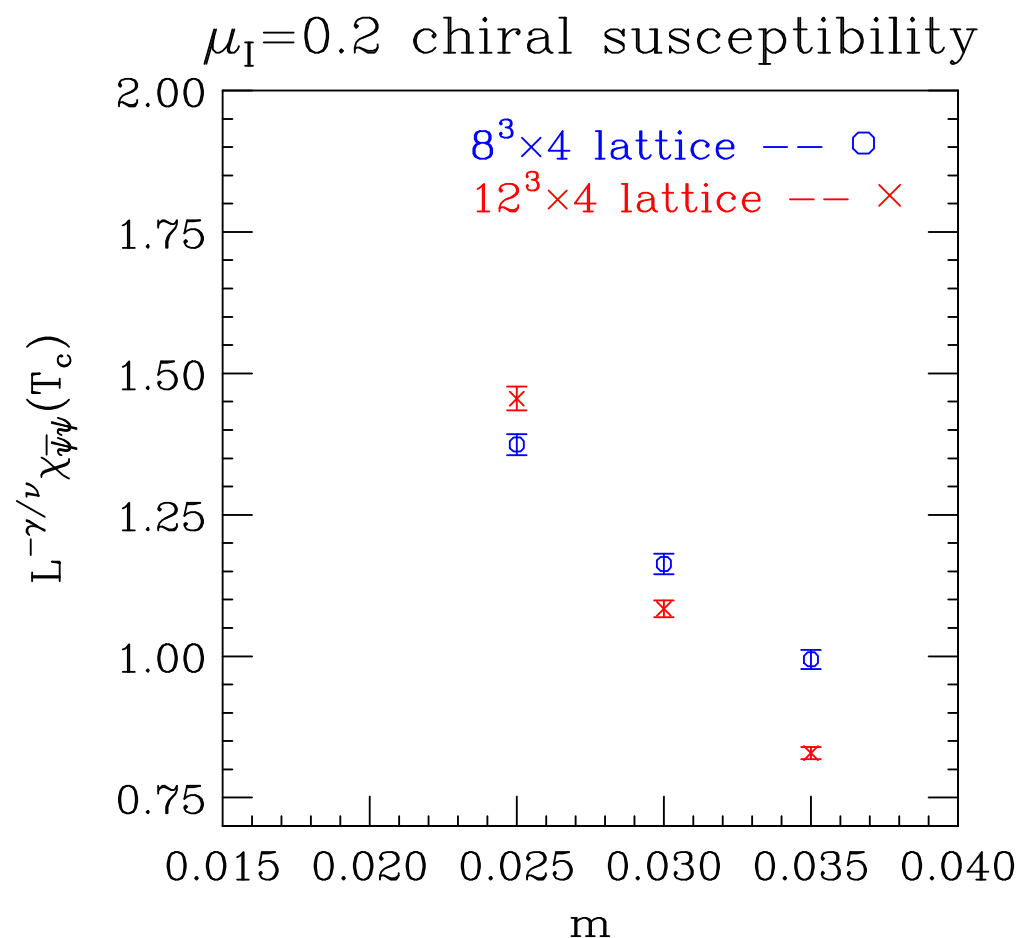

Figure 3: Finite size scaling for the peak of the chiral susceptibilities at $\mu_{I}=0.2$

\section{Equation of state}

The equation-of-state (EOS) expresses the pressure $p$, the entropy density $s$ and the energy 
density $\varepsilon$ as functions of temperature $T$ and $\mu_{I}$. (Calculations of the equation of state for QCD at finite $T$ and $\mu$ have been performed for example in [6] and [7].) The pressure $p$ is simply related to the partition function through:

$$
p=\frac{T}{V} \ln Z\left(T, \mu_{I}\right)
$$

However, we do not actually measure the partition function $Z$ in our simulations, only observables. $Z(T, 0)$ can be calculated by numerically integrating

$$
\frac{d \ln Z}{d \beta}=\left\langle 6 \frac{V}{T} S_{g}\right\rangle
$$

where $S_{g}$ is the plaquette action. We can then numerically integrate

$$
\frac{d \ln Z}{d \mu_{I}}=\left\langle\frac{N_{f}}{8} \frac{V}{T} j_{0}^{3}\right\rangle
$$

at constant $\beta$, where $\frac{N_{f}}{8} j_{0}^{3}$ is the the isospin density, to obtain $Z\left(T, \mu_{I}\right)$. Figure 4 shows the $\mu_{I}$ dependence of $j_{0}^{3}$ at fixed $\beta$ values. For the upper $2 \beta$ s we also have 'data' for larger $\mu_{I} \mathrm{~s}$, up to saturation which occurs for $\mu_{I} \approx 2$.

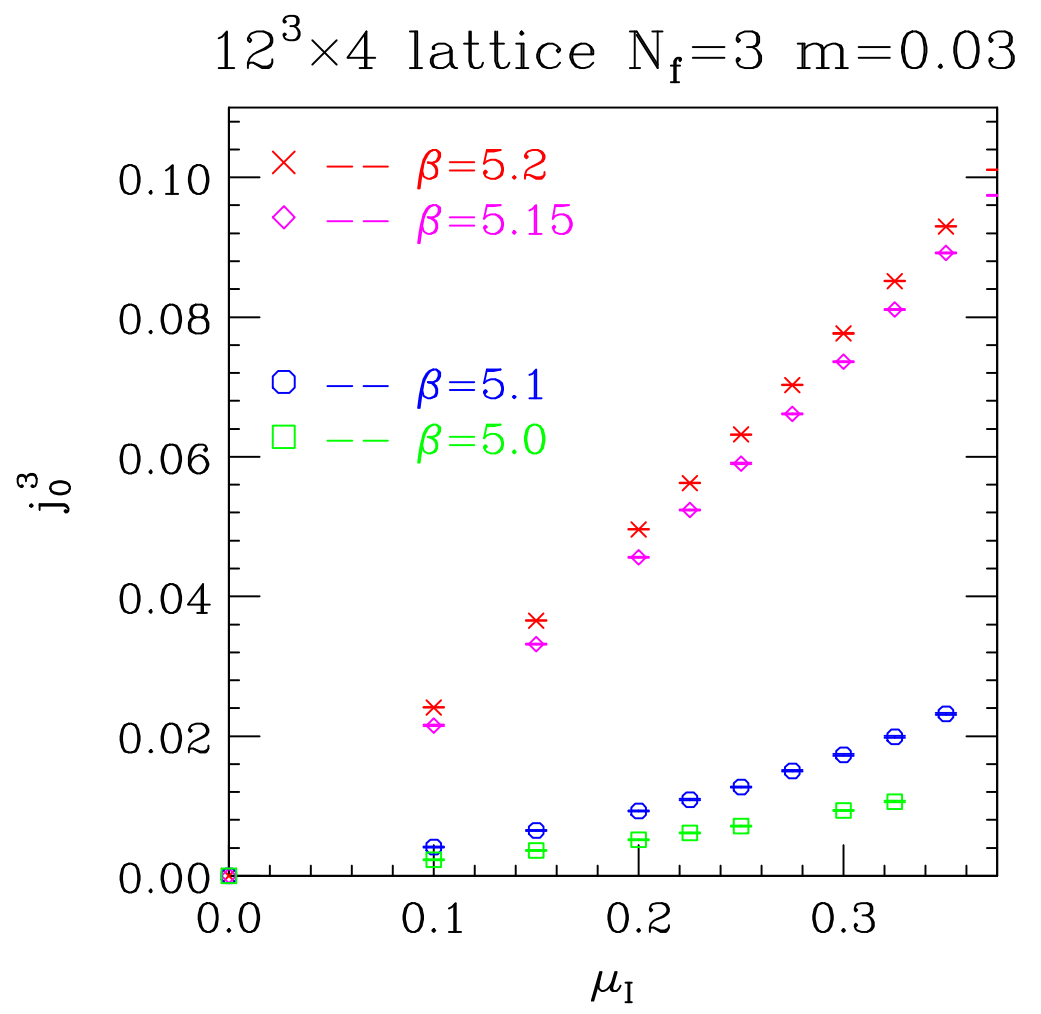

Figure 4: Isospin density as functions of $\mu_{I}$ at fixed $\beta$ values

To obtain $T$ in physical units requires knowledge of the running of the coupling constant, $\beta=\beta(a)$. This is determined at $\mu_{I}=0$. Once this running of the coupling constant is known, this can be used to determine $\varepsilon$, since

$$
\varepsilon=\frac{T^{2}}{V} \frac{\partial}{\partial T} \ln Z
$$




\section{Discussion and conclusions}

For $\mu$ small enough or $T$ large enough that phase-quenched QCD is in its normal rather than its superfluid phase, full QCD and phase-quenched QCD are expected to have the same phase structure. Lattice simulations indicate that the fluctuations of the phase of the fermion determinant, on lattices large enough to observe this phase structure, are sufficiently small for this to be so [8]. Random matrix calculations in the epsilon regime agree with this conclusion [9].

For 3-flavour lattice QCD our phase-quenched simulations show no evidence for a critical endpoint in the range of $m$ and $\mu$ values where it would have been expected if it were associated with the critical point observed when the quark mass is varied. This is in agreement with the results of de Forcrand and Philipsen obtained using analytic continuation methods from simulations at imaginary $\mu$, for full QCD [10].

We suggest that the softening of the transition as $\mu_{I}$ is increased is because the introduction of an isospin chemical potential reduces the symmetry (at least for even numbers of flavours). Reducing symmetry tends to soften transitions. For example, reducing the number of flavours from 3 to 2 , reduces the symmetry at $m=0, \mu=0$ from $S U(3) \times S U(3)$ to $S U(2) \times S U(2)$ and the transition softens from first order to second. The addition of a mass breaks chiral symmetry to a pure vector symmetry, and softens phase transitions to crossovers.

Determination of the true scaling field for the magnetic order parameter - a linear combination of the chiral condensate, the plaquette action and the isospin density - is needed to remove the finite size effects. Use of the methods of de Forcrand and Philipsen to improve the signal/noise in calculating the $\mu^{2}$ dependence of the Binder cumulants, would also help [11, 12].

Fodor and Katz have predicted a critical endpoint at $T_{E}=162(2) \mathrm{MeV}$ and $\mu=120(13) \mathrm{MeV}$, using their reweighting methods [13]. However, this $\mu$ is considerably beyond $m_{\pi} / 2$ and thus beyond the reach of both phase-quenched and analytic-continuation methods.

We are performing simulations along lines of constant $\beta$ to determine the equation-of-state for phase-quenched QCD to compare with that for full QCD $[6,7,14]$. At low $\beta$ s we are restricted to $\mu_{I}<m_{\pi}$. At high $\beta \mathrm{s}$, where the system is in the plasma phase for all $\mu_{I} \mathrm{~s}$, we can cover the whole range of $\mu_{I}$. What can we learn from the resonance gas model [15] and chiral perturbation theory about this equation-of-state?

\section{Acknowledgements}

DKS was supported in part by US Department of Energy contract DE-AC-02-06CH11357. JBK is supported in part by a National Science Foundation grant NSF PHY03-04252. We thank Philippe de Forcrand and Frithjof Karsch for helpful discussions. Simulations were performed on the Jazz cluster at Argonne, Tungsten, Cobalt and Copper at NCSA, Bassi and Jacquard at NERSC, DataStar at SDSC and PCs in Argonne's HEP Division.

\section{References}

[1] M. A. Clark and A. D. Kennedy, Phys. Rev. D 75 (2007) 011502 [arXiv:hep-lat/0610047].

[2] J. B. Kogut and D. K. Sinclair, Phys. Rev. D 74 (2006) 114505 [arXiv:hep-lat/0608017]. 
[3] D. K. Sinclair and J. B. Kogut, PoS LAT2006 (2006) 147 [arXiv:hep-lat/0609041].

[4] K. Binder, ’ Z. Phys. B 43 (1981) 119.

[5] A. M. Ferrenberg and R. H. Swendsen, Phys. Rev. Lett. 61, 2635 (1988).

[6] S. Ejiri, F. Karsch, E. Laermann and C. Schmidt, Phys. Rev. D 73 (2006) 054506 [arXiv:hep-lat/0512040].

[7] F. Csikor, G. I. Egri, Z. Fodor, S. D. Katz, K. K. Szabo and A. I. Toth, JHEP 0405 (2004) 046 [arXiv:hep-lat/0401016].

[8] C. R. Allton et al., Phys. Rev. D 66 (2002) 074507 [arXiv:hep-lat/0204010].

[9] K. Splittorff and J. J. M. Verbaarschot, Phys. Rev. D 75 (2007) 116003 [arXiv:hep-lat/0702011].

[10] P. de Forcrand and O. Philipsen, JHEP 0701 (2007) 077 [arXiv:hep-lat/0607017].

[11] P. de Forcrand, parallel talk presented at this meeting.

[12] O. Philipsen, parallel talk presented at this meeting.

[13] Z. Fodor and S. D. Katz, JHEP 0404 (2004) 050 [arXiv:hep-lat/0402006].

[14] C. R. Allton, S. Ejiri, S. J. Hands, O. Kaczmarek, F. Karsch, E. Laermann and C. Schmidt, Phys. Rev. D 68 (2003) 014507 [arXiv:hep-lat/0305007].

[15] F. Karsch, K. Redlich and A. Tawfik, Phys. Lett. B 571 (2003) 67 [arXiv:hep-ph/0306208]. 